Universidade Tecnológica Federal do Paraná - UTFPR

Campus Ponta Grossa - Paraná - Brasil

ISSN: 1981-3686/v. 08, n. 01: p. 1154-1164, 2014

D.O.I.:10.3895/S1981-36862014000100002
Revista Brasileira deTecnologia

Agroindustrial

\title{
ELABORAÇÃO DE BOLO DE MEL ENRIQUECIDO COM FIBRAS DOBAGAÇO DA INDÚSTRIA CERVEJEIRA
}

\section{DEVELOPMENT OF CAKE HONEY ENRICHED WITH THE FIBERBAGASSE BREWING INDUSTRY}

\author{
Nathalie Hamine Panzarini ${ }^{1}$; Andrea Rabbers ${ }^{1}$; José Luiz Ferreira da Trindade ${ }^{1}$; Eloiza Aparecida Silva Avila \\ de Matos ${ }^{1}$ Maria Helene Giovanetti Canteri ${ }^{1}$; Juliana Vitoria Messias Bittencourt ${ }^{1}$ \\ ${ }^{1}$ Universidade Tecnológica Federal do Paraná - UTFPR - Ponta Grossa - Brasil \\ nathalie_h.p@hotmail.com
}

\begin{abstract}
Resumo
A indústria cervejeira, ao longo de seu processamento, gera grandes quantidades de resíduo. Um dos mais descartados é o da matéria prima utilizada para a preparação do mosto cervejeiro, conhecido como bagaço de cerveja. Esse resíduo constituído por um alto teor fibras é destinado em sua grande maioria para a alimentação animal. As fibras alimentares vêm despertando interesse de especialistas das áreas de nutrição e saúde por seus benefícios à saúde. Foi utilizado mel nas formulações por também ser um alimento com propriedades benéficas à saúde. O consumo de mel no Brasil é considerado baixo comparado a outros países e a utilização do mesmo nas formulações possibilita o aumento de seu consumo com uma nova opção, além da "in natura", a forma mais consumida. Esse trabalho teve como objetivo elaborar um produto destinado a alimentação humana à base de bagaço de cerveja e mel, bem como promover sua avaliação e estimar o nível de aceitabilidade. Foram elaboradas duas formulações finais, com 7\% e 10,5\% de bagaço de cerveja. O bagaço de cerveja utilizado apresentou $40 \%$ de fibras. Ambas as formulações de bolo de mel enriquecido com fibras classificaram-se dentro dos padrões microbiológicos tornando-se seguros ao consumo. $O$ indice de aceitabilidade (IA\%) foi maior que 80\% nos atributos: impressão global, cor, aroma, textura e sabor. Com os resultados obtidos espera-se valorização do resíduo, aumentando seu valor agregado e conseqüentemente, trazendo benefícios para indústria cervejeira.
\end{abstract}

Palavras-chave: Bagaço de cerveja. Mel; Alimento funcional; Bolo de mel; Fibras.

\section{1. .Introdução}

O mel é considerado um fluido viscoso, aromático e doce elaborado por abelhas a partir do néctar e/ou exsudatos sacarínicos de plantas, principalmente de origens florais, os quais, depois de levados para a colméia pelas abelhas, são amadurecidos por elas e estocados no favo para sua alimentação (BRASIL, 2000).

O crescente mercado dos produtos naturais, aliado ao interesse dos consumidores na prevenção de doenças, tem pressionado a indústria alimentícia na busca por produtos mais saudáveis e direcionado pesquisas nesse sentido. Neste contexto, os alimentos funcionais ganharam destaque pelos efeitos benéficos que promovem à saúde (ANJO, 2004). 
De acordo com a ANVISA, o alimento ou ingrediente que alegar propriedades funcionais, além de atuar em funções nutricionais básicas, irá desencadear efeitos benéficos à saúde e deverá ser também seguro para o consumo sem supervisão médica (ANVISA, 2010).

Da mesma forma que aconteceu com a indústria, a evolução do agronegócio e o desenvolvimento dos processos de transformação de alimentos levaram à geração de muitos resíduos, sendo que estes são um dos principais problemas ambientais, não só do Brasil, mas do mundo como um todo (GIORDANO, 2000).Mudanças no processamento e a crescente exigência do consumidor por alimentos com qualidade sensorial, nutricional e que tragam benefícios à saúde incentivam o estudo de novos ingredientes para a indústria de alimentos (IDRIS et al., 1996; MOSCATTO, PRUDENCIOFERREIRA e HAULY, 2004).

O bagaço da cerveja é o resíduo resultante do processo inicial da fabricação de cervejas. Este bagaço provém do processo de obtenção do mosto, pela fervura do malte moído e dos adjuntos, que após a filtração, resulta num resíduo que atualmente é destinado para ração animal (AQUARONE, 2001). Esse resíduo contém fibras e proteínas que ajudam no funcionamento do organismo (SOUZA, 2003).

Pelo fato de o resíduo ser gerado em grande quantidade durante a produção da cerveja, e também por ser rico em fibras e proteínas, outras formas de utilização, além da alimentação animal, podem ser estudadas, como seu uso na alimentação humana (ASSIS et al., 2006).

\footnotetext{
O bolo é tão antigo, quanto o hábito humano de celebrar acontecimentos sociais com alimentos. O fascínio levou por essa massa basicamente constituída de farinha, ovos e açúcar levou a se multiplicar através dos tempos e povos, elaborando receitas com diferentes ingredientes, conferindo-lhe sabor e textura. Embora a alta proporção de açúcar, amido e gordura favoreçam uma contribuição calórica, o bolo, pela sua propriedade de aceitar diversos ingredientes na confecção, constituiu fontes inesgotáveis de opções de enriquecimentos nutritivo (ROSSETTI, 2006).
}

Os bolos prontos para o consumo vêm adquirindo crescente importância no mercado de produtos de panificação no Brasil. A qualidade dos bolos é determinada por características essenciais, como: textura macia, que deve permanecer inalterada ao longo da vida de prateleira do produto; superfície uniforme; homogeneidade do miolo; volume adequado; palatabilidade e sabor agradável; e facilidade de processamento (PAVANELLI; CICHELLO; PALMA, 2010).

Bolo é o produto assado, preparado à base de farinhas ou amidos, açúcar, fermento químico ou biológico, podendo conter leite, ovos, manteiga, gordura e outras substancias alimentícias que caracterizam o produto (ANVISA, 2011).O presente artigo teve como objetivo elaborar uma formulação de bolo para alimentação humana com resíduo similar ao da indústria cervejeira e mel. 


\section{Material e Métodos}

\section{Material}

Os ingredientes para a formulação do bolo foram adquiridos em mercado de Ponta Grossa - PR. O bagaço, resultante da fase inicial do processo de fabricação de cervejas, foi obtido em aula prática de bebidas para fermentação de cerveja artesanal, na UTFPR, Câmpus Ponta Grossa.

\section{Métodos}

\section{Elaboração das Formulações}

Inicialmente várias formulações foram testadas no Laboratório de Panificação. Levando em conta características organolépticas como cor, textura e aroma, e sabor, as mais adequadas formulações foram produzidas e avaliadas nesse trabalho.

Formulação I $\rightarrow$ com $7 \%$ de bagaço de cerveja;

Formulação II $\rightarrow$ com $10,5 \%$ de bagaço de cerveja.

A Tabela 1 discrimina as matérias-primas e o respectivo percentual utilizado nas formulações utilizadas para a elaboração do bolo de mel enriquecido com fibras.

Tabela 1 - Formulações do Bolo de Mel enriquecido com Fibras

\begin{tabular}{ccc}
\hline Ingredientes & $\begin{array}{c}\text { Formulação I } \\
(\boldsymbol{\%})\end{array}$ & Formulação II (\%) \\
\hline Farinha de trigo & 26 & 22,5 \\
Achocolatado em pó & 5,5 & 5,5 \\
Açúcar & 8 & 8 \\
Mel & 11 & 11 \\
Canela em pó & 0,5 & 0,5 \\
Bicarbonato de sódio & 0,5 & 0,5 \\
Fermento químico & 0,5 & 0,5 \\
Leite & 33 & 33 \\
Ovo & 8 & 8 \\
Bagaço da cerveja & 7 & 10,5 \\
Total & 100 & 100 \\
\hline
\end{tabular}

As formulações foram submetidas ao processo térmico ao mesmo tempo para efeitos de comparação.

\section{Análise granulométrica do Bagaço da Cerveja}

A análise granulométrica foi realizada para a determinação do tamanho das partículas do bagaço da cerveja sendo utilizado um equipamento vibratório (Granutest) composto por 13 peneiras, cujas aberturas variaram de $150 \mu \mathrm{m}$ a $1,7 \mathrm{~mm}$, onde se colocou $87 \mathrm{~g}$ do bagaço em agitação a $5 \mathrm{~Hz}$ por 10 minutos, anotando-se a quantidade de amostra retida em cada peneira e o diâmetro médio foi calculado por Diâmetro médio de Sauter utilizando a fórmula: 


$$
\bar{D}_{s}=\frac{1}{\sum_{i=1}^{n} \frac{x_{i}}{d_{p i}}} \quad, \quad X_{i}=\frac{M_{r}}{M_{T}}
$$

$\mathrm{M}_{\mathrm{r}}$ - massa retida;

$\mathrm{M}_{\mathrm{T}}$ - massa total;

$\mathrm{D}_{\mathrm{pi}}-$ diâmetro médio entre as peneiras com massa retida.

\section{Análises Físico-Químicas do Bolo de Mel Enriquecido com Fibras}

No produto acabado, foram feitas em triplicata, as análises descritas abaixo.

Análise instrumental de cor: Avaliaram-se as coordenadas de cor eletronicamente, utilizando UltranScanPRO da marca HunterLab, no modo R Tran sensor D65 ângulo de $10^{\circ}$. As cores das amostras dos bolos foram avaliadas através de três parâmetros, o CIE L* $\mathrm{a}^{*} \mathrm{~b}^{*}$ (Comissão Internacional de Iluminantes). O parâmetro $\mathrm{L}^{*}$ define a luminosidade $\left(\mathrm{L}^{*}=0\right.$ preto e $\mathrm{L}^{*}=100$ branco) e $\mathrm{a}^{*}$ e $\mathrm{b}^{*}$ são responsáveis pela cromaticidade $\left(+\mathrm{a}^{*}\right.$ vermelho e $-\mathrm{a}^{*}$ verde,$+\mathrm{b}^{*}$ amarelo e b* azul). Para cada tratamento, as determinações foram efetuadas em triplicata e os resultados expressos como média (MCGUIRE, 1992).Atividade de água: As amostras foram inseridas no aparelho AquaLab 4TE. B

\section{Análises Microbiológicas do Bolo de Mel Enriquecido com Fibras}

Segundo a Resolução - CNNPA n 12 , de 1978, da ANVISA, para produto com designação "bolo", foram realizadas tais análises microbiológicas:Coliformes totais;Coliformes termotolerantes; Clostrídios sulfito redutores (a $44^{\circ} \mathrm{C}$ ); Staphylococus aureus; Salmonella sp; Bolores e leveduras.

\section{Análise Sensorial}

Para avaliar as características dos bolos foram preparadas amostras das duas formulações do bolo e aplicado um teste de aceitação para avaliar os atributos: cor, aroma, textura, sabor e impressão global, utilizando escala hedônica de 1 a 7 pontos, considerando desgostei muitíssimo e gostei muitíssimo respectivamente (ABNT, NBR 14141, 1998).

O teste foi realizado no Laboratório de Análise Sensorial da Universidade Tecnológica Federal do Paraná - campus Ponta Grossa, por um painel sensorial não treinado formado por 40 pessoas, de diferentes idades, entre eles, estudantes, funcionários e professores da universidade. As amostras foram devidamente oferecidas em guardanapos, juntamente com água, com uma ficha de avaliação e uma bala.

O índice de Aceitabilidade foi calculado através da expressão matemática de Meilgaard, Civille, Carr (1991): 
$\mathrm{IA} \%=\underline{\mathrm{X} \cdot 100}$

$\mathrm{N}$

Onde: $\mathrm{X}=$ média de cada amostra

$\mathrm{N}$ = nota máxima, de cada amostra, dada pelos provadores

\section{Análise Estatística}

Foram calculadas as médias \pm desvio-padrão.A análise estatística foi realizada utilizandose o programa SASM - Agri (CANTERI et al., 2001). Os dados referentes à composição centesimal e às diferentes frações da fibra alimentar dos produtos analisados foram comparados por ANOVA, seguida do teste de Tukey, a $5 \%$ de probabilidade de erro $(\mathrm{p}<0,05)$.

\section{RESULTADOS E DISCUSSÃO}

\section{Análise granulométrica do bagaço da cerveja}

O peneiramento teve como objetivo melhorar a aparência e patabilidade dos produtos. Na realização da análise granulométrica, o bagaço peneirado foi submetido a 13 peneiras para determinar seu diâmetro médio. As médias retidas nas peneiras com abertura de $1,40 \mathrm{~mm}$ e $250 \mu \mathrm{m}$, $212 \mu \mathrm{m}$ e $180 \mu \mathrm{m}$ e, $1,70 \mathrm{~mm}$ e $500 \mu \mathrm{m}$ não apresentaram diferença significativa $(\mathrm{p}<0,05)$. Isso comprova que o bagaço peneirado apresenta grande variação no tamanho de suas partículas. $\mathrm{O}$ diâmetro médio calculado foi de $637 \mu \mathrm{m}$. A Figura 1 indica a porcentagem de massa das partículas retidas em cada peneira.

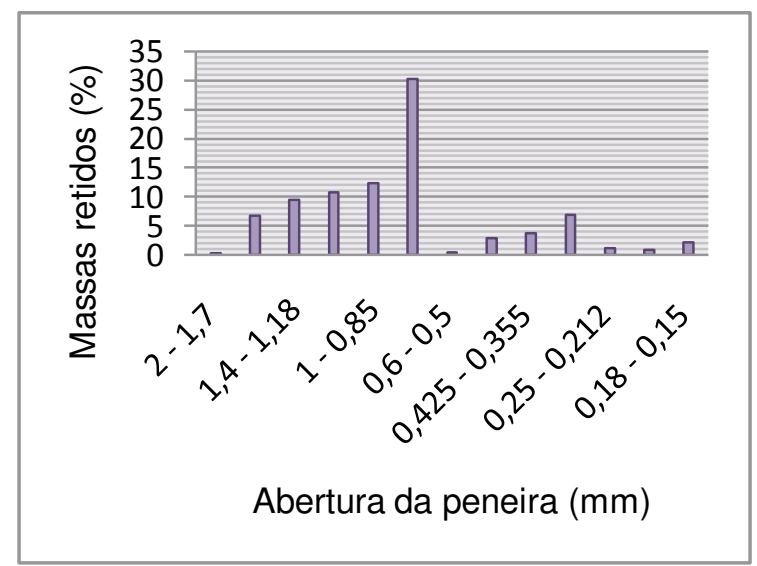

Figura 1 - Porcentagem de massa das partículas retidas em cada peneira

A maior porcentagem do resíduo se situa entre $1,18 \mathrm{~mm}$ e $600 \mu \mathrm{m}(71,49 \%)$ sendo que $34,38 \%$ ficou retida na peneira com $600 \mu \mathrm{m}$ de abertura. Segundo a Instrução Normativa $\mathrm{n}^{\circ} 8$, de 2 de junho de 2005 (MAPA, 2005), 95\% do produto deve passar pela peneira com abertura de malha de $250 \mu \mathrm{m}$ para se classificar com o farinha de trigo, sendo que o bagaço teve apenas 4,62\%. 
A mistura dos ingredientes para a elaboração do produto apresentou aspecto pastoso e o produto elaborado apresentou cor visualmente mais escura comparada ao bolo de mel tradicional por conter o bagaço da cerveja. Os resultados médios das análises físico-químicas estão apresentados na Tabela 2.

Tabela 2 - Características físico-químicas do Bolo de Mel enriquecido com Fibras

\begin{tabular}{cccc}
\hline Amostra & $\begin{array}{c}\text { Umidade } \\
(\boldsymbol{\%})\end{array}$ & $\begin{array}{c}\text { Cinzas } \\
(\boldsymbol{\%})\end{array}$ & Fibras (\%) \\
\hline Formulação I (7\%) & $32,08^{\mathrm{a}}( \pm 0,028)$ & $1,7^{\mathrm{a}}( \pm 0,005)$ & $9^{\mathrm{a}}( \pm 0,01)$ \\
Formulação II $(10,5 \%)$ & $32,84^{\mathrm{b}}( \pm 0,017)$ & $2,3^{\mathrm{b}}( \pm 0,015)$ & $11^{\mathrm{b}}( \pm 0,01)$ \\
\hline
\end{tabular}

Letras diferentes na mesma coluna indicam diferença estatística pelo teste de Tukey (95\% de significância)

Houve diferença significativa entre as amostras no teor de umidade, cinzas e fibras.Maior teor de umidade foi encontrado no bolo elaborado com maior porcentagem do bagaço, portanto, com mais fibra alimentar, o que se justifica pela propriedade das fibras de reterem e manterem água em sua estrutura durante o processo de cocção. Valores de umidade semelhantes aos encontrados para os bolos analisados no presente estudo foram citados por Ferreira, Oliveira e Pretto (2001). Oliveira e Reyes (1990) e Souza et al. (2000) verificaram elevação na umidade de biscoitos à medida que o teor de fibra era aumentado.

Os valores de análise obtidos, descritos na Tabela 2 estão coerentes com o esperado. A perda e o ganho de umidade vão ocorrer continuamente, de uma região para outra, como forma de equilíbrio dinâmico entre os componentes e o meio (LABUZA et al., 1998).

Nas formulações elaboradas no presente trabalho, as médias foram mais altas, com 1,7\% para formulação I e 2,3\% para formulação II, comprovando o alto teor de fibra do bagaço da cerveja (Tabela 2). Segundo a legislação vigente, resolução RDC no 360, de 23 de dezembro de 2003 (ANVISA, 2003) e portaria $\mathrm{n}^{\circ}$ 27, de 13 de janeiro de 1998 (ANVISA, 1998), pode-se dizer que as duas formulações de bolo com de bagaço de cerveja classificam-se como alimento com alto teor ou rico em fibras por apresentarem valor médio de $10 \mathrm{~g} / 100 \mathrm{~g}$ de produto, visto que o mínimo preconizado pela Legislação para ser produto com alto teor de fibras é $6 \mathrm{~g} / 100 \mathrm{~g}$ de produto.

Análise de Cor

As amostras foram lidas diretamente no aparelho no modo refractância, utilizando-se a escala $L^{*}, a^{*}, b^{*}\left(\right.$ CIELAB) em que L* é a luminosidade, $a^{*}$ a intensidade do vermelho/verde e $b^{*}$ a intensidade do amarelo/azul. Os resultados estão apresentados na Tabela 3.Em relação aos parâmetros de cromaticidade ( $\mathrm{a}^{*} \mathrm{e} \mathrm{b}^{*}$ ), foi possível afirmar que as duas amostras se apresentaram nas regiões vermelha e amarelo já que a leitura do colorímetro demonstrou valores positivos para estas coordenadas. Silva (2007) menciona que a combinação dos cromos positivos a* e b* resulta 
na coloração marrom, cor característica de produtos elaborados com chocolate e seus derivados. No caso, o bolo foi elaborado com achocolatado em pó.

Tabela 3 - Valores obtidos do parâmetro L* e das coordenadas cromáticas a* e b* da análise de cor das amostras de bolo

\begin{tabular}{lcccc}
\hline \multicolumn{1}{c}{ Amostra } & $\mathbf{L}^{*}$ & $\mathbf{a}^{*}$ & $\mathbf{b}^{*}$ \\
\hline Formulação I $(7 \%)$ & $36,45^{\mathrm{a}}( \pm 0,461)$ & $6,51^{\mathrm{a}}( \pm 0,16)$ & $7,19^{\mathrm{a}}( \pm 0,31)$ \\
Formulação II $(10,5 \%)$ & $35,78^{\mathrm{a}}( \pm 0,093)$ & $6,42^{\mathrm{a}}( \pm 0,08)$ & $6,98^{\mathrm{a}}( \pm 0,096)$ \\
\hline \multicolumn{6}{l}{} \\
Letras iguais na mesma coluna indicam igualdade estatística pelo teste de Tukey $(95 \%$ de significância)
\end{tabular}

Quanto ao parâmetro de luminosidade $\left(\mathrm{L}^{*}\right)$, as amostras foram consideradas escuras, já que na escala de 0 a 100 apresentaram valores abaixo de $50\left(\mathrm{~L}^{*}<50\right)$ (COHEN; JACKIX, 2005). As amostras não apresentaram diferença significativa $(\mathrm{p}<0,05)$, porém a formulação I apresentou-se mais clara, devido à menor porcentagem de bagaço de cerveja utilizado, considerando-a com maior luminosidade.

Outros fatores podem ter afetado a coloração dos bolos. A presença de açúcares e ovos, assim como o calor, acelera reações de caramelização e Maillard, levando ao escurecimento progressivo da crosta e do miolo (GIESE, 2000).

\section{Atividade de Água}

A atividade de água indica a quantidade de água disponível para realizar o movimento molecular e suas transformações, e promover o crescimento microbiano no produto. Os resultados da Aw dos bolos estão apresentados na Tabela 4.

Tabela 4 - Avaliação da Aw das amostras de Bolo

\begin{tabular}{lccc}
\hline \multicolumn{1}{c}{ Amostra } & \multicolumn{1}{c}{ Aw } & Temperatura $^{\mathbf{0}} \mathbf{C}$ \\
\hline Formulação I $(7 \%)$ & $0,9066^{\mathrm{a}}( \pm 0,0004)$ & 25,04 \\
Formulação II $(10,5 \%)$ & $0,8952^{\mathrm{b}}( \pm 0,0008)$ & 25,03 \\
\hline
\end{tabular}

Letras diferentes na mesma coluna indicam diferença estatística pelo teste de Tukey (95\% de significância)

A atividade de água média dos bolos foi de 0,9. Segundo Jardim \& Germer (1997), valores acima de 0,80 e 0,88 favorecem o desenvolvimento de bolores e leveduras, respectivamente.

Bassinello et al. (2010) na elaboração de bolos com diferentes proporções de farinhas de arroz e de feijão, encontraram em média 0,90 para atividade de água analisada no primeiro dia para todos os tratamento. Valores semelhantes aos encontrados no presente trabalho. 
Para avaliação microbiológica das amostras foi seguido a Resolução - CNNPA n ${ }^{\circ} 12$, de 1978 (ANVISA, 1978) para Produtos de confeitaria com designação bolo para Coliformes a 35²C/g, Clostrídio sulfito redutores $\left(\mathrm{a} 44^{\circ} \mathrm{C}\right)$ e Bolores e leveduras e a Resolução - RDC $\mathrm{N}^{\mathrm{o}} 12$, de 2 de Janeiro de 2001 (ANVISA, 2001) para bolachas e biscoitos, sem recheio, com ou sem cobertura, incluindo pão de mel, cookies e similares para Coliformes a $45^{\circ} \mathrm{C} / \mathrm{g}$, Estafilococos coagulase positiva/g e Salmonella sp/ $25 \mathrm{~g}$. Os resultados estão na Tabela 5.

Tabela 5 - Características microbiológicas do Bolo de Mel Enriquecido com Fibras

\begin{tabular}{|c|c|c|c|c|}
\hline Análises & Formulação & Formulação & \multicolumn{2}{|c|}{$\overline{\text { Padrão exigido }}$} \\
\hline Coliformes a $35^{\circ} \mathrm{C} / \mathrm{g}$ & $<10 \mathrm{UFC} / \mathrm{g}$ & $<10 \mathrm{UFC} / \mathrm{g}$ & $10^{2} / \mathrm{g}(\operatorname{Max})$ & $\mathrm{CNNPA} \mathrm{n}^{\circ} 12$, de 1978 \\
\hline Coliformes a $45^{\circ} \mathrm{C} / \mathrm{g}$ & $<10 \mathrm{UFC} / \mathrm{g}$ & $<10 \mathrm{UFC} / \mathrm{g}$ & 10 & $\mathrm{RDC} \mathrm{N}^{\circ} 12$, de 2001 \\
\hline $\begin{array}{l}\text { Clostrídios sulfito } \\
\text { redutores }\left(\mathrm{a} 44^{\circ} \mathrm{C}\right)\end{array}$ & $<10 \mathrm{UFC} / \mathrm{g}$ & $<10 \mathrm{UFC} / \mathrm{g}$ & $10^{2} / \mathrm{g}(\mathrm{Max})$ & $\mathrm{CNNPA} \mathrm{n}^{\circ} 12$, de 1978 \\
\hline Estaf.coag.positiva/g & $<10 \mathrm{UFC} / \mathrm{g}$ & $<10 \mathrm{UFC} / \mathrm{g}$ & $5 \times 10^{2}$ & $\mathrm{RDC} \mathrm{N}^{\circ} 12$, de 2001 \\
\hline Bolores e leveduras & $9 \times 10$ & $6 \times 10^{2}$ & $10^{3} / \mathrm{g}(\operatorname{Max})$ & $\mathrm{CNNPA} \mathrm{n}^{\circ} 12$, de 1978 \\
\hline Salmonella sp/25g & Ausência & Ausência & Ausência em 25g & RDC No 12, de 2001 \\
\hline
\end{tabular}

Conforme a Tabela 5, as duas formulações estão dentro do padrão estabelecido, tornandose seguros para o consumo, garantindo sua qualidade.

\section{Análise sensorial}

As amostras foram avaliadas conforme os atributos de cor, aroma, textura, sabor e a nota global. Foi adicionada ao bolo, uma cobertura de chocolate para melhorar a avaliação sensorial.

A Tabela 6 mostra as médias e o índice de Aceitabilidade (IA\%) da análise sensorial das duas formulações do bolo de mel.

Tabela 6 - Médias e Índice de Aceitabilidade (IA\%) da Análise Sensorial

\begin{tabular}{ccccc}
\hline \multirow{2}{*}{ Atributo } & \multicolumn{2}{c}{ Formulação I (7\%) } & \multicolumn{2}{c}{ Formulação II (10,5\%) } \\
& Média & IA (\%) & Média & IA (\%) \\
\hline Cor & $5,63^{\mathrm{a}}$ & 80,36 & $5,70^{\mathrm{b}}$ & 81,43 \\
Aroma & $5,85^{\mathrm{a}}$ & 83,57 & $5,70^{\mathrm{b}}$ & 81,43 \\
Textura & $5,65^{\mathrm{a}}$ & 80,71 & $5,80^{\mathrm{b}}$ & 82,86 \\
Sabor & $5,93^{\mathrm{a}}$ & 84,64 & $6,03^{\mathrm{b}}$ & 86,14 \\
Impressão global & $6,18^{\mathrm{a}}$ & 88,29 & $6,18^{\mathrm{a}}$ & 88,29 \\
\hline
\end{tabular}

Letras diferentes na mesma linha indicam diferença estatística pelo teste de Tukey (95\% de significância)

O critério de decisão utilizado para o índice ser de boa aceitação foi igual ou superior a 70\%. Dessa forma, segundo a tabela 9, as formulações I e II apresentaram um bom índice de 
aceitabilidade para todos os atributos avaliados sendo que o atributo de impressão global foi o de maior aceitabilidade atingindo, em ambas as formulações, 88,29\%.

O índice de aceitabilidade a partir de $70 \%$ indica que o produto pode ser avaliado em testes de mercado com boas probabilidades de obter sucesso. $\mathrm{O}$ índice de Aceitabilidade foi realizado para justificar o uso da escala hedônica de 7 pontos.

A Formulação I teve uma maior aceitabilidade apenas no atributo aroma, com 83,57\% em comparado com a formulação II. Já Mattos (2010), obteve maior aceitação com 88,88\% no atributo sabor, no desenvolvimento de pão de forma com $30 \%$ de bagaço de malte.

Moscattoet al. (2004) observaram que os atributos cor, textura e impressão global não foram afetados pela adição destes ingredientes na formulação, quando comparados à aceitação sensorial da formulação padrão na aceitação sensorial de bolos de chocolate adicionados de farinha de yacon e inulina.

A Tabela 7 mostra a quantidade de votos de cada escala para o atribulo impressão global. Os atributos cor, textura e sabor, $81,43 \%, 82,86 \%, 86,14 \%$, respectivamente, tiveram um maior índice de aceitabilidade na formulação II, apresentando diferença estatística $(\mathrm{p}<0,05)$.

Embora a formulação II possua uma maior quantidade do bagaço da cerveja em sua formulação, apresentou, mesmo assim, avaliação sensorial positiva. Isso contraria a ideia de que produtos com alto teor de fibras são menos aceitos pelos consumidores.

Tabela 7 - Quantidade de votos de cada escala para o atribulo Impressão Global

\begin{tabular}{ccc}
\hline & Impressão Global & \\
\hline Escala & Formulação I & Formulação II \\
\hline $7-$ Gostei muitíssimo & 16 & 17 \\
$6-$ Gostei muito & 15 & 16 \\
$5-$ Gostei & 9 & 5 \\
$4-$ Não gostei / nem desgostei & & 1 \\
$3-$ Desgostei & & \\
$2-$ Desgostei muito & \\
1 - Desgostei muitíssimo &
\end{tabular}

Os provadores no geral demonstraram curiosidade em relação à utilização do bagaço da cerveja para enriquecimento de fibras no produto, e muitos elogiaram a iniciativa de reaproveitar esse resíduo no desenvolvimento de novos produtos.

\section{CONCLUSÃO}

A partir dos resultados pode-se concluir que os produtos elaborados tiveram uma boa aceitabilidade sensorial para os atributos: impressão global, cor, aroma, textura e sabor, indicando que os produtos podem seguir para testes de mercado, com grande probabilidade de fazer sucesso. 
O sabor foi o segundo atributo que teve mais aceitabilidade, isso mostra que os produtos não ficaram com o sabor característico do bagaço da cerveja e que os consumidores estão mais preocupados com a qualidade do produto em relação ao gosto, um dos atributos que mais satisfaz o ser humano.

As duas formulações se enquadraram no padrão microbiológico, o que torna o alimento seguro e garante a qualidade para os consumidores.

O produto se classificou como rico em fibras e pode ser uma ótima opção de alimento, com propriedades funcionais, também por conter mel em sua formulação. Sendo as fibras provenientes do bagaço da cerveja, tem-se uma nova opção para o destino desse resíduo, preservando o meio ambiente, o que favorece economicamente a indústria cervejeira e torna-se uma inovação por agregar valor ao produto. Esse resultado proporciona também o incentivo na ingestão de mel, substância que traz tantos benefícios à saúde da população.

\begin{abstract}
The beer industry over its processing, generates huge amounts of waste, one of the most discarded is the raw material used for the preparation of mash brewer, known as bagasse beer. This residue consists of a high fiber content is intended mostly for animal feed. The dietary fiber shave attracted experts from the fields of nutrition and health for their benefits, and they help prevent several diseases, and the environmental problems caused by industrial waste every day are worrying the population, and environmental control have become stricter on this issue, we decided to develop a quality product with the use of bagasse Beer, becoming a food with functional properties. Honey was used in formulations for food with also beneficial health properties. The use of honey in Brazil is considered low compared to other countries, its use in the formulation enables the increase of their consumption with a new option, besides the "natural", the most consumed. This study aimed to develop a product intended for human consumption based on bagasse of beer and honey as well as promote its assessment and estimate the level of acceptability. Two final formulations were prepared with $7 \%$ and10.5\% of bagasse beer. The beer bagasse used showed40\% fibers and products made can better med as "high fiber", by submitting more than $6 \%$ of fibers in its composition. Both formulations of honey cake enriched with fiber were classified with microbiological standards making its consumption. The acceptability index (AI\%) was higher than $80 \%$ in the attributes:the overall impression, color,aroma,textureand flavor. With the results expected recovery of the waste, increasing its value and consequently bringing benefits for the brewing industry.
\end{abstract}

Keywords: Bagasse of beer. Honey. Functional food. Honey cake. Fibers.

\title{
REFERÊNCIAS
}

ABNT. ABNT, NBR 14141, 1998. Disponível em <http://pt.scribd.com/doc/11757612/Analise-Sensorial-deAlimentos-Capitulo-6> Acesso em 23 ago.2012.

ANJO, D. F. C. Alimentos funcionais em angiologia e cirurgia vascular. Disponível em:<http://www.jvascbr.com.br/04-03-02/04-03-02-145/04-03-02-145.pdf>. Acesso em: 17 fev. 2011.

ANVISA - Agência Nacional da Vigilância Sanitária. Disponível em: <http://portal.anvisa.gov.br/> Acesso em: 21 fev. 2011.

- Rotulagem de alimentos. Disponivel em: <http://www.anvisa.gov.br/rotulo/>. Acesso em: 25 mai. 2011.

- Resolução - CNNPA no 12, de 1978 . Disponivel em http://www.anvisa.gov.br/legis/resol/12_ 78.pdf> Acesso em: 23 ago.2012.

- Resolução - RDC No 12, de 2 de Janeiro de 2001. Disponível em <http://www.anvisa.gov.br/legis/ resol/12_01rdc.htm> Acesso em: 23 ago. 2012. 
ASSIS, L. M; MEDINA, A. L; CARVALHO, D; RODRIGUES, R. Elaboração de Farelo a Partir de Bagaço de Malte de Cevada Proveniente da Indústria Cervejeira. XV Congresso de Iniciação Científica - VIII Encontro de Pós-Graduação - 2006. Disponível em: <http://www.ufpel.edu.br/cic/2006/resumo_expandido/CA/CA_00959.pdf>. Acesso em: 18 fev. 2011.

BASSINELLO, Priscila Zaczuk; CARVALHO, Rosangela Nunes; ALMEIDA, Raquel Pereira de; ARAÚJO, Marcela Rios; COBUCCI, Rosário de Maria Arouche. Desenvolvimento de Mistura para Bolo com Farinhas de Quirera de Arroz e Bandinha de Feijão. Comunicado técnico - Embrapa. Disponível em:<http://www.cnpaf.embrapa.br/publicacao/comunicadotec/comt_193.pdf>. Acesso em: 12 mar. 2011.

BRASIL. Ministério da Agricultura, Pecuária e Abastecimento. Anexo IV. Requisitos mínimos para determinação do valor de cultivo e uso de feijão (Phaseolus vulgaris), para a inscrição no registro nacional de cultivares - RNC. Disponível em: <http://www.agricultura.gov.br>. Acesso em: 7 abr. 2011.

CANTERI, M. G., Althaus, R. A., VIRGEnS Filho, J. S., Giglioti, E. A., GOdOY, C. V. SASM-Agri: Sistema para análise e separação de médias em experimentos agrícolas pelos métodos Scoft - Knott, Tukey e Duncan. Revista Brasileira de Agrocomputação, v. 1, n. 2, p. 18-24. 2001.

CERQUEIRA, P. M. de. Avaliação da farinha de semente de abóbora (Cucúrbita máxima, L.) no trato intestinal e no metabolismo glicídico e lipídico em ratos. 68 p. Dissertação (Mestrado em Ciência e Tecnologia de Alimentos), Instituto de Tecnologia, Universidade Federal Rural do Rio de Janeiro - UFRRJ, Seropédica, 2006.

COHEN, K. O.; JACKIX, M. N. H. Estudo do liquor de cupuaçu. Revista Ciência e Tecnologia de Alimentos, v. 25, n. 1, 2005.

FERREIRA, S. M. R.; OLIVEIRA, P. V.; PRETTO, D. Parâmetros de qualidade do pão francês. Boletim CEPPA, v. 19, n. 2, p. 301-318, 2001.

GIORDANO, S. R. Gestão ambiental no sistema agroindustrial. In: ZYLBERSZTAJN, D.; NEVES, M.F. (Orgs.). Economia e gestão de negócios agroalimentares: indústria de alimentos, indústria de insumos, produção agropecuária, distribuição. São Paulo: Pioneira, 2000. p.255-281.

HISTÓRIA DO BOLO - História da Culinária, 2011. Disponível em<http://www.fleischmann.com.br/culinaria/default.asp?page=http://www.fleischmann.com.br/culinaria/dicas_do_pad eirito/curiosidades_interna.asp?id=399>. Acesso em: 15 mar. 2011.

INSTITUTO ADOLFO LUTZ. Normas Analíticas do Instituto Adolfo Lutz. v. 1: Métodos químicos e físicos para análise de alimentos, 3. ed. São Paulo: IMESP, 1985. p. 27.

LABUZA, T.P.; HYMAN, C.R. Moisture migration and control in multi-domain foods. Trends Food Science and Technoogy., Amsterdan, v.9, p.47-55, 1998

JARDIM, D.C.P.; GERMER, S.P.M. Atividade de águaemalimentos, Campinas: Ital, 1997. p. 3-2.

MAPA. Instrução Normativa $\mathbf{n}^{\mathbf{0}} \mathbf{8}$, de $\mathbf{2}$ de junho de $\mathbf{2 0 0 5}$ Disponível em <http://www.claminas. com.br/pdf/Farinha_de_trigo.pdf> Acesso em : 23ago.2012.

MATTOS, Camila. Desenvolvimento de um pão fonte de fibras a partir do bagaço de malte. Monografia. Universidade Federal do Rio Grande do Sul. Porto Alegre, 2010. Disponível em: <http//www.lume.ufrgs.br/handle/10183/28403 ->. Acesso em: 30 abr. 2011.

MOSCATTO, J. A.; PRUDÊNCIO-FERREIRA, S. H.; HAULY, M. C. O. Farinha de yacon e inulina como ingredientes na formulação de bolo de chocolate. Ciência e Tecnologia de Alimentos, Campinas, v. 24, n. 4, p.634640, 2004.http://dx.doi.org/10.1590/S0101-20612004000400026

McGUIRE, R. G. Reporting of objective color measurements. Horticultural Science, v. 27, n. 12, p. 1254-1555, 1992.

OLIVEIRA, S. P.; REYES, F. G. R. Biscuits with a high content of corn fibre: preparation, chemical and technological characterization, and acceptability. Ciência e Tecnologia de Alimentos, v. 10, n. 2, p. 273-286, 1990.

PAVANELli, A. P.; CICHELlO, M. S.; PALMA, E. J. Emulsificantes como agentes de Aeração em Bolos. Artigo Técnico- 2000. Disponível em:

$<$ http://www.oxiteno.com.br/aplicacoes/mercados/doc/documento.asp?artigotecnico=4\&segmento=0100\&idioma=PO\& $\mathrm{r}=$.pdf>. Acesso em: 11 mai. 2011.

ROSSETTI. Farinha de trigo1999. Disponível em:

<http:/www.Rossetti.et.br/discurse/detalhe.asp?vine=6evfim=6evcodigo=2149>. Acesso em: 10 mar. 2011.

SOUZA, M. L. et al. (sic). Processamento de cookies de castanha-do-Brasil. In: CONGRESSO BRASILEIRO DE CIÊNCIA E TECNOLOGIA DE ALIMENTOS, 17., 2000, Ceará. Livros de resumos... Ceará, 2000. v. 3 , p. 11.

Submetido em 03 set. 2012, Aceito para publicação em 06 out. 2013. 